\title{
Survival and amplification of germ like cells isolated from mouse ES cell formed embryoid bodies in recipient seminiferous tubules
}

\author{
Guanghui Cui ${ }^{1}$, Weiwei Zheng ${ }^{1}$, Chao Zhang ${ }^{1}$, Xin Guo ${ }^{1}$, Jie Qin ${ }^{1}$, Zhiming Cai ${ }^{1}$, Xigu Chen ${ }^{1}$ \\ ${ }^{1}$ Laboratory of Male Reproduction, Peking University Shenzhen Hospital, Shenzhen, Guangdong 518036, China
}

Recent studies demonstrated that germ cells could be isolated from mouse embryonic stem cell formed embryoid body, and currently there is no report indicates that the isolated germ cells can survive and initiate spermatogenesis in seminiferous tubules.

In this study, we planed to isolate germ like cells from embryoid bodies prepared from mouse EGFP-ES cells, after retinoic acid treatment, the cells would be transplanted into seminiferous tubules of busulfan-treated mice, aiming to see if these cells could initiate amplification and differentiation in the recipient seminiferous tubules. Results showed that after being treated with retinoic acid for six days, alkaline phosphatase expressing cells could still be found in embryoid body derived cells, indicating the existence of retinoic acid-resistant primordial germ cells. When the SSEA- $1^{+}$cells isolated from embryoid bodies were stimulated with retinoic acid for six days, some of these cells expressed cell markers of $\mathbf{H s p 9 0}-\alpha$, integrin $\beta 1$ and $\alpha 6$, resembling the expression profile of spermatogonial stem cells. Forty-five days after cell transplantation, a little amount of GFP-expressing cells attached to the basement membrane of seminiferous tubule and formed small colonies; Three months later, these cells started amplification in the form of cell chains with varied length, and moving towards the lumen of the seminiferous tubules. There was no GFP-expressing cells existed in non-cell-transplanted seminiferous tubules. In our study, although no obvious spermatogenesis was observed, all the results indicated that the embryoid body contains germ like cells, and these cells can survive and initiate amplification in recipient seminiferous tubules.

Keywords: embryonic stem cell, embryoid body, retinoic acid, seminiferous tubule, transplantation, spermatogenesis Cell Research (2008) 18:s44. doi: 10.1038/cr.2008.134; published online 4 August 2008

Correspondence: Xigu Chen

E-mail: cgh1968@tom.com 